\title{
OBSERVATIONS ON THE OCCURRENCE AND MATURATION OF SPINITECTUS INERMIS (NEMATODA: CYSTIDICOLIDAE) IN THE SOUSA RIVER, PORTUGAL
}

\author{
Aurélia Saraiva, Anabela Pereira and Cristina Cruz
}

Departamento de Zoologia e Antropologia, Faculdade de Ciências, Universidade do Porto, Praça Gomes Teixeira, 4099-002 Porto, Portugal

Spinitectus inermis (Zeder, 1800) is a rare specific nematode of the European eel, Anguilla anguilla (L.), that ranges widely in Europe (North, Baltic and Mediterranean Seas basins) but occurs only locally, particularly in lakes, fishponds and in the lowland reaches of rivers (Moravec F. 1977: Folia Parasitol. 24: 85; Moravec F. 1979: Acta Soc. Zool. Bohemoslov. 43: 35-42; Moravec F. 1994: Parasitic Nematodes of Freshwater Fishes of Europe. Academia, Prague, 473 pp.). This nematode has been studied since the beginning of the $19^{\text {th }}$ century but its morphology was little known until the redescription by Moravec (1979, op. cit.) plus additional data revealed by scanning electron microscopy (Moravec F. 1996: Parasite 3: 169-174). The knowledge of the biology of this nematode is also scarce. Recently, however, it was found that nymphs of several species of mayflies (Ephemeroptera) were suitable experimental intermediate hosts of this nematode (Saraiva A.M., Moravec F., Pereira A., Cruz C. 2002: Folia Parasitol. 49: 118-126). Since S. inermis is an abundant parasite of eels in the Sousa River, Portugal, its seasonal occurrence and maturation were followed and the results are presented herein.

The population dynamics of $S$. inermis was followed in eels from the lowest reaches of the Sousa River, a tributary of the Douro River, from the locality of Senhora do Salto, situated about $20 \mathrm{~km}$ east of Porto, northern Portugal. Monthly samples were taken by electrofishing from November 1998 to October 1999 (total sample size 326; mean body length of fish $22.0 \pm 4.42 \mathrm{~cm})$. Fish were examined for parasites and the recovered nematodes were washed in physiological saline $(0.9 \%)$, fixed in hot $70 \%$ ethanol, stored in $70 \%$ ethanol and cleared in glycerol for light microscopical examination for sex and maturity stage determination.

The occurrence of nematodes, sex ratio and stages of development of females (gravid females with mature eggs / females in other developmental stages) throughout the year were compared by Chi-square test (Siegel S., Castellan N.J. Jr. 1989: Nonparametric Statistics for the Behavioral Sciences. McGraw-Hill Book Company, New York, 399 pp.).

The prevalence and mean intensity of infection of $S$. inermis in eels during the studied period is shown in Fig. 1. $S$. inermis was present throughout the study period. Significant difference in the occurrence of this parasite $\left(\chi^{2}=32.81\right.$, d.f. $=$ $11, \mathrm{p}<0.05)$ was found during the year. Prevalence reached high values $(30-57 \%)$ from November to February and low values $(10 \%$ and $13 \%)$ in June and July. Intensity ranged from 1 to 26 . The high values of mean intensity occurred in January (9) and June (14), with the minimum value (2) in December. Adults (males and females) of $S$. inermis occurred in eels

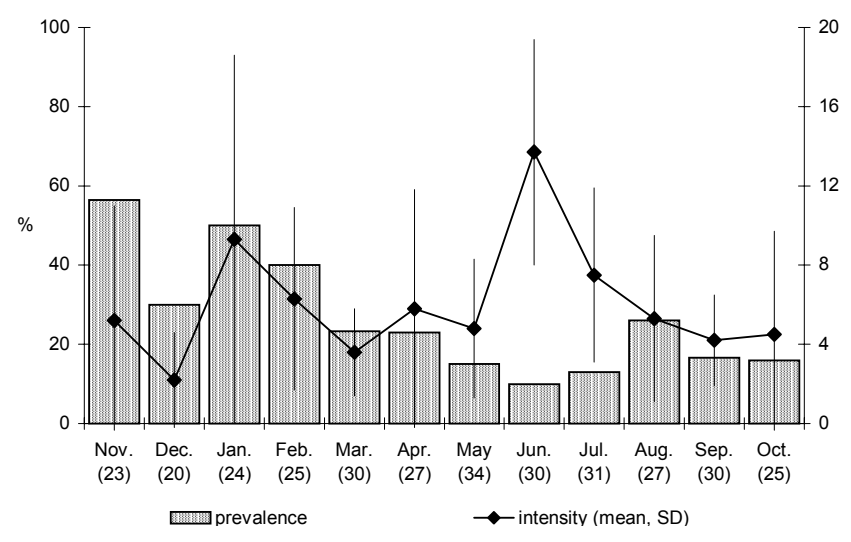

Fig. 1. Monthly changes in the prevalence and intensity of Spinitectus inermis in eels from the Sousa River from November 1998 to October 1999. Fish sample size in parentheses.

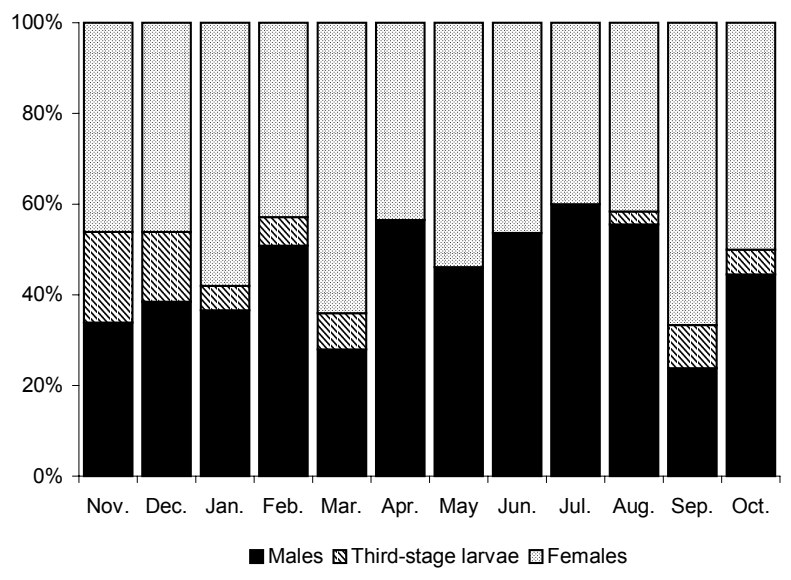

Fig. 2. Monthly changes in the percentage of males, females and third-stage larvae of Spinitectus inermis in eels from the Sousa River from November 1998 to October 1999.

throughout the year (Fig. 2). No significant difference occurred in the proportion of males and females during the year $\left(\chi^{2}=14.8\right.$, d.f. $\left.=11, p>0.05\right)$. Undifferentiated nematodes (third-stage larvae) occurred from November to March and from August to October (Fig. 2), and high monthly percentage was observed in November and December. The monthly percentage of females with mature eggs, females with immature eggs, and females without eggs (fourth-stage larvae 


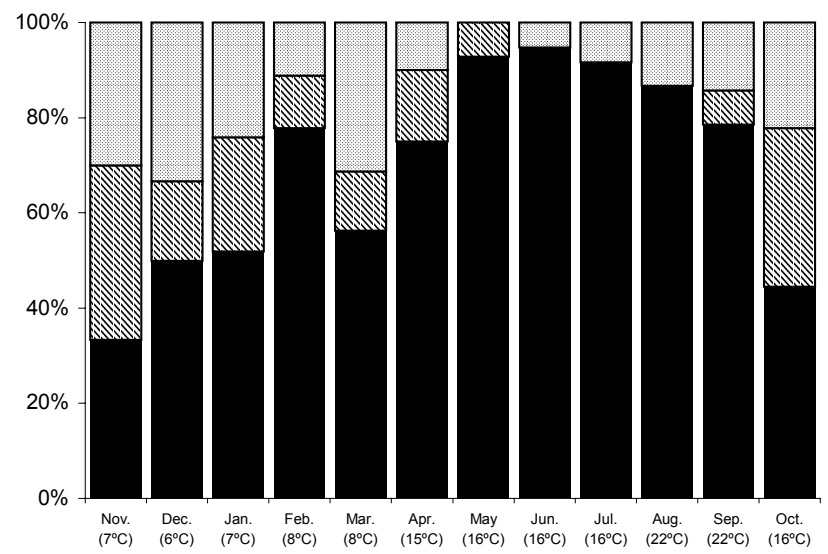

- females with mature eggs $\mathbb{\$}$ females with immature eggs $\mathbf{0}$ females without eggs

Fig. 3. Monthly changes in the percentage of females with mature eggs, females with immature eggs and females without eggs of Spinitectus inermis in eels from the Sousa River from November 1998 to October 1999. Water temperature in parentheses.

and juveniles) during the year are shown in Fig. 3. A significant difference was detected in the monthly percentage of females with mature eggs and females in all other developmental stages $\left(\chi^{2}=21.44\right.$, d.f. $\left.=11, p<0.05\right)$ during the year. Females with mature eggs were found throughout the year, in high percentages from May to September (79-95\%) and in low percentage in November (33\%). Females without eggs or with immature eggs were not detected in May and from June to August, respectively. High percentage of females without eggs or with immature eggs was observed from October to January (48-67\%).

In the present study the prevalence of $S$. inermis reached maximum values in late autumn and during winter, coinciding with the main recruitment, as indicated by the high percentage of third-stage larvae observed during this period of the year. The main reproductive period of $S$. inermis in the Sousa River occurred in late spring and summer when water temperature was high $\left(16-22^{\circ} \mathrm{C}\right)$ and favourable for the growth of the nematode. The decrease of prevalence in this period seems to be related to the death of parasites and their expulsion from the host after copulation (males) and oviposition (females). The absence of third-stage larvae during this period (from April to July) appears to be caused by scarcity of infected intermediate hosts during this period of the year. The high intensities of $S$. inermis infection in eels could be associated with high intensities of infection in the intermediate host population. Eggs of $S$. inermis are provided with denticulate polar caps (Saraiva et al. 2002, op. cit.) that seem to have an adhesive function and could cause aggregation of several eggs during oviposition and their intake by a single intermediate host. Saraiva et al. (2002, op. cit.) stated that several species of mayfly nymphs could serve as experimental intermediate hosts of this nematode.

The profile of the occurrence and the maturation of Rhabdochona denudata (Dujardin, 1845), nematodes that have mayfly nymphs as intermediate hosts, in barbel, Barbus bocagei Steindachner (reported as B. barbus bocagei), from several rivers in Léon, NW Spain (Pereira Bueno J.M., Alvarez Pelliteiro M.P. 1979: An. Fac. Vet. Léon 25: 155-198) was very similar to that reported in the present study. According to these authors the values of prevalence of $R$. denudata were maximal in autumn and winter, coinciding with the main recruitment, and minimal in summer. Furthermore, no larva was found in the majority of spring and summer months, and females with mature eggs, observed during all the year, occurred especially during spring and summer.

One other species of Spinitectus Fourment, 1883, Spinitectus gordoni Cordero del Campillo et Alvarez Pelliteiro, 1976, is reported from European freshwater fishes (Moravec 1994, op. cit.), it is restricted to trouts from rivers of northwestern Spain (Léon). This species is quite abundant (prevalence $40 \%$, mean intensity 19) and the highest rates of infection occur in autumn (Cordero del Campillo M., Alvarez Pelliteiro M.P. 1976: Rev. Iber. Parasitol. 36: 35-66), which is similar to what was observed in the present study of $S$. inermis. These authors recorded two heavily infected trouts (709 and 924 nematodes) with very few adults and many larvae (third- and fourth-stage) but the season when these fishes were caught was not recorded. Since Léon and northern Portugal (both located in the northern part of the Iberian Peninsula) have similar climatic conditions and once it can be supposed that similarly to $S$. inermis, mayfly nymphs may be the intermediate host of $S$. gordoni (Moravec 1994, op. cit.), both species may have a similar pattern of seasonal occurrence and maturation.

It seems, from the above discussion, that the abiotic and biotic factors that occur in Iberian Peninsula provide optimal conditions for the life cycles of the species of Spinitectus that occur in fresh waters in Europe. 